\title{
Influence of spherical anisotropy on the optical properties of plasmon resonant metallic nanoparticles
}

\author{
Y.X. Ni · D.L. Gao $\cdot$ Z.F. Sang $\cdot$ L. Gao $\cdot$ C.W. Qiu
}

Received: 7 June 2010 / Accepted: 12 October 2010 / Published online: 3 November 2010

(c) Springer-Verlag 2010

\begin{abstract}
We demonstrate the features of the dipole and quadrupole resonant modes in extinction spectra of spherically anisotropic nanoparticles based on full-wave scattering theory. It is found that in a core-shell nanosphere, the introduction of spherical anisotropy in the core leads to a blue shift in resonant wavelengths for small nanoparticles, while, for large nanoparticles (core radius larger than $90 \mathrm{~nm}$ ), the dipole resonant wavelength remains unchanged with the variation of spherical anisotropy. In addition, the peak strengths for the dipole and quadrupole modes are also studied. Numerical simulations show that the strong localization of electric fields can be further enhanced and tuned by adjusting the spherical anisotropy in the core. In contrast, the anisotropy introduced in the shell results in a blue shift for small nanoparticles but a red shift for nanoparticles with larger size. The tunability of plasmon resonant shifts in extinction spectra and tailored localization of enhanced fields are revealed.
\end{abstract}

\section{Introduction}

The interaction of light with metallic nanoparticles has been an active subject of both experimental and theoretical research for decades, because these nanoparticles pos-

Y.X. Ni · D.L. Gao · Z.F. Sang · L. Gao (凶)

Jiangsu Key Laboratory of Thin Films, Department of Physics, Soochow University, Suzhou 215006, China

e-mail: leigao@suda.edu.cn

\section{C.W. Qiu}

Department of Electrical and Computer Engineering, National University of Singapore, 4 Engineering Drive 3, Singapore

117576, Singapore

e-mail: eleqc@nus.edu.sg sess unique optical, electric and magnetic properties compared to bulk materials. For instance, when the incident frequency equals the inherent surface plasmon frequency of the nanoparticles, surface resonance takes place and both the enhancement of local fields in the surface of the nanoparticles and complicated far-field spectra were predicted [1, 2]; high tunability of the surface plasmon resonant frequency just by varying the core-shell radius ratio was also realized $[3,4]$. Furthermore, the core-shell microstructure provides an alternative topology for manipulating electromagnetic fields at nanoscale dimension [5-9]. As a consequence, the coreshell structure was extensively explored for many potential applications such as sensor applications [7], optical communication [10] and biomedical applications [5, 11].

On the other hand, the study of electromagnetic interaction with anisotropic core-shell particles has attracted much attention owing to its promising applications in scattering enhancement or suppression, various nano-antennas and novel optical devices [12-17]. In general, two types of anisotropies exist in natural and artificially engineered materials: Cartesian anisotropy and spherical anisotropy [18]. For the particles with Cartesian anisotropy, an analytical method based on multipole expansion was established to characterize the wave propagation by extending Mie theory in the isotropic case to the uniaxial case [19]. Here, we concentrate on spherical anisotropy, which means that the permittivity (or permeability) tensor is diagonal in spherical coordinates, and the dielectric element along the radial direction differs from the one along the tangential direction [20]. Actually, spherical anisotropy was indeed found in many nanostructures such as biological cells containing mobile charges and real phospholipid vesicles [21-24], and it is quite important for the creation of the electromagnetic cloaking of invisibility [25]. Note that spherical anisotropy is quite different from Cartesian anisotropy in that spheri- 
cal anisotropy means that the permittivity tensor is locally anisotropic and depends on the positions $(x, y, z)$, while Cartesian anisotropy indicates that the permittivity tensor is overall anisotropic and is independent of the positions. More recently, it was found that spherical anisotropy plays an important role in achieving the enhancement of nonlinear susceptibilities for second- and third-harmonic generation and controlling resonant frequencies [26]. Furthermore, the suitable adjustment of spherical anisotropy of the coreshell particle can also result in a tunable electromagnetic cloaking [13]. In addition, the near-field and far-field optical properties of coated particles with metallic core and spherically anisotropic shell [2] or with metallic shell and spherically anisotropic core [27] have already been investigated, which are only valid under the electrostatic condition, i.e. the quasistatic limit.

Motivated by the core-shell particles and the peculiarity of spherical anisotropy, we study the extinction properties of core-shell nanoparticles of two types: (1) a spherically anisotropic core with a noble-metal shell and (2) a spherically anisotropic shell with a noble-metal core. Based on modified Mie scattering theory for the structure with spherical anisotropy [1, 15], we investigate the extinction properties of core-shell nanospheres with spherical anisotropy based on full-wave electromagnetic theory instead of a quasistatic approximation as in [2]. We reveal the dependence of the optical properties of dipole and quadrupole plasmon resonant modes and field localization upon the degree of spherical anisotropy as well as the size of the nanoparticles.

\section{Theoretical model}

We consider a nonmagnetic coated spherical particle consisting of a spherically anisotropic core or anisotropic shell (see Fig. 1), which is illuminated by a polarized plane wave with unit amplitude $E_{i}=\hat{e}_{x} \mathrm{e}^{\mathrm{i} k_{1} z}$, where $k_{1}=\omega / c \sqrt{\varepsilon_{1}}\left(\varepsilon_{1}\right.$ is the relative permittivity of the medium). For nonmagnetic

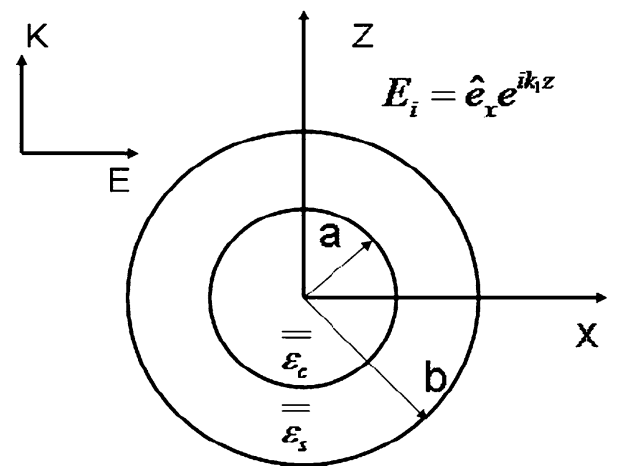

Fig. 1 Geometry of the scattering of a plane wave by a core-shell spherical nanoparticle immersed in a dielectric host medium particles, the relative permeability $\mu$ is equal to 1 . The relative permittivity tensors of the core $\left(\overline{\bar{\varepsilon}}_{\mathrm{c}}\right)$ and the shell $\left(\overline{\bar{\varepsilon}}_{\mathrm{s}}\right)$ are written as in spherical coordinates,

$\overline{\bar{\varepsilon}}_{\mathrm{c}}=\left(\begin{array}{ccc}\varepsilon_{\mathrm{cr}} & 0 & 0 \\ 0 & \varepsilon_{\mathrm{ct}} & 0 \\ 0 & 0 & \varepsilon_{\mathrm{ct}}\end{array}\right), \quad \overline{\bar{\varepsilon}}_{\mathrm{s}}=\left(\begin{array}{ccc}\varepsilon_{\mathrm{sr}} & 0 & 0 \\ 0 & \varepsilon_{\mathrm{st}} & 0 \\ 0 & 0 & \varepsilon_{\mathrm{st}}\end{array}\right)$,

where $\varepsilon_{i \mathrm{r}}$ (or $\varepsilon_{i \mathrm{t}}$ ) represents the permittivity elements in a direction parallel (perpendicular or tangential) to the local $r$ axis for $i=\mathrm{c}$ or $\mathrm{s}$.

With Maxwell's equations, the local electric and magnetic fields in the core and shell can be expressed as

$$
\begin{aligned}
& \nabla \times \mathbf{H}=-\mathrm{i} \omega \overline{\bar{\varepsilon}}_{\mathrm{c}(\mathrm{s})} \mathbf{E}, \\
& \nabla \times \mathbf{E}=\mathrm{i} \omega \mu_{0} \mathbf{H},
\end{aligned}
$$

where the time-dependent factor $\mathrm{e}^{-\mathrm{i} \omega t}$ is suppressed. Based on scattering theory $[28,29]$, the scattered wave can be regarded as a superposition of TE and TM waves, and we can obtain the scattering coefficients in free space, i.e. $A_{n}^{\mathrm{TM}}$ and $A_{n}^{\mathrm{TE}}[13]$,

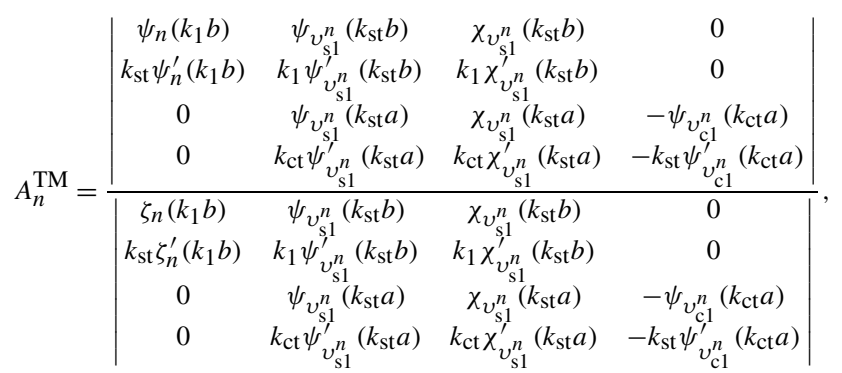

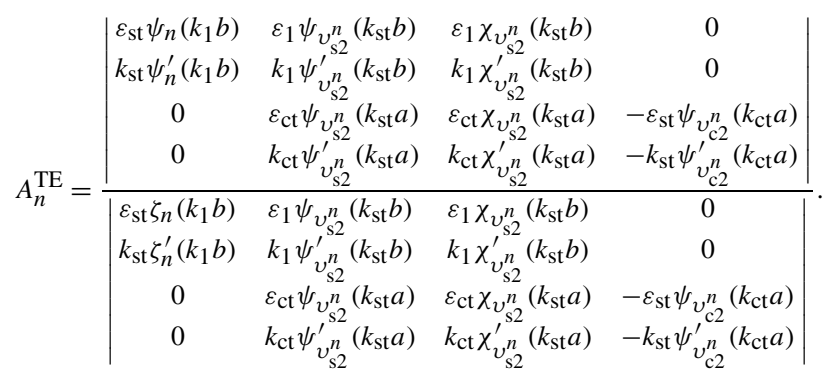

The detailed elaborations of (3) and (4) are provided in the Appendix. Note that for nonmagnetic particles, $A_{n}^{\mathrm{TM}}$ is much more important than $A_{n}^{\mathrm{TE}}$.

The extinction cross section of the coated sphere is defined as

$$
C_{\mathrm{ext}}=\frac{2 \pi}{k_{1}^{2}} \sum_{n=1}^{\infty}(2 n+1) \operatorname{Re}\left(A_{n}^{\mathrm{TE}}+A_{n}^{\mathrm{TM}}\right) .
$$


On the other hand, for small coated particles, the electrostatic solution is enough. In the long-wavelength and lowfrequency limit $\left(k_{1} b \rightarrow 0\right.$ and $\left.k_{i t} b \rightarrow 0\right)$, the main contribution to the extinction cross section results from the electric dipole $A_{1}^{\mathrm{TM}}$. In this connection, $A_{1}^{\mathrm{TM}}$ is related to the electric dipole factor $D$ with the form $A_{1}^{\mathrm{TM}}=2 / 3 \mathrm{i}\left(k_{1} b\right)^{3} D$, where $D$ can be simplified as

$D=\frac{\left(\epsilon_{\mathrm{sr}} v_{\mathrm{s} 1}^{1}-\varepsilon_{1}\right)\left[\epsilon_{\mathrm{cr}} v_{\mathrm{c} 1}^{1}+\epsilon_{\mathrm{sr}}\left(1+v_{\mathrm{s} 1}^{1}\right)\right]+\left(\frac{a}{b}\right)^{\left(2 v_{\mathrm{s} 1}^{1}+1\right)}\left[\varepsilon_{1}+\left(1+v_{\mathrm{s} 1}^{1}\right) \epsilon_{\mathrm{sr}}\right]\left(\epsilon_{\mathrm{cr}} v_{\mathrm{c} 1}^{1}-\epsilon_{\mathrm{sr}} v_{\mathrm{s} 1}^{1}\right)}{\left(\epsilon_{\mathrm{sr}} v_{\mathrm{s} 1}^{1}+2 \varepsilon_{1}\right)\left[\epsilon_{\mathrm{cr}} v_{\mathrm{c} 1}^{1}+\epsilon_{\mathrm{sr}}\left(1+v_{\mathrm{s} 1}^{1}\right)\right]+\left(\frac{a}{b}\right)^{\left(2 v_{\mathrm{s} 1}^{1}+1\right)}\left[2 \varepsilon_{1}-\left(1+v_{\mathrm{s} 1}^{1}\right) \epsilon_{\mathrm{sr}}\right]\left(\epsilon_{\mathrm{sr}} v_{\mathrm{s} 1}^{1}-\epsilon_{\mathrm{cr}} v_{\mathrm{c} 1}^{1}\right)}$.

Correspondingly, the extinction cross section $C_{\text {ext }}$ can be expressed as

$C_{\mathrm{ext}}=\frac{\omega}{c} \operatorname{Im}(\alpha)+\frac{1}{6 \pi}\left(\frac{\omega}{c}\right)^{4}|\alpha|^{2}$,

where $\alpha=4 \pi b^{3} D$ is the electric polarizability. Here we would like to mention that for small coated particles, in which the electrostatic limit is valid, one can adopt (7) to calculate the extinction cross section [27]. However, when the coated particle is large, one must resort to the full-wave electromagnetic solution (5).

For simplicity, we assume that the metallic material is gold $(\mathrm{Au})$, whose relative permittivity is described by the Drude formula [30]

$\varepsilon_{\mathrm{Au}}=1-\frac{\omega_{\mathrm{p}}^{2}}{\omega^{2}+\mathrm{i} \omega \gamma}+10.3$,

where $\omega_{\mathrm{p}}\left(=1.37 \times 10^{16} \mathrm{~s}^{-1}\right)$ and $\gamma$ are the bulk plasma frequency and modified collision frequency, respectively. $\gamma$ is expressed as $\gamma=\left(1.07 \times 10^{14}+v_{\mathrm{f}} / w\right) \mathrm{s}^{-1}$, where $v_{\mathrm{f}}(=$ $1.4 \times 10^{6} \mathrm{~m} / \mathrm{s}$ ) is the Fermi velocity and $w$ is the size of the particle. For instance, if the core is made of $\mathrm{Au}, w$ equals the radius of the core $a$ and, if the shell is made of $\mathrm{Au}, w$ should be written as $b-a$ ( $b$ is the radius of the shell). In this paper, we assume that $b=1.5 a$. In addition, the background medium is assumed to be water with $\varepsilon_{1} \approx 1.77 \varepsilon_{0}$.

\section{Numerical results}

In what follows, we perform numerical calculations of the extinction cross section under different core-shell structures and different sizes. We aim at discussing the tunable resonant shifts in the extinction properties and tailorable field enhancement caused by the spherical anisotropy. Full-wave simulations are also provided to illustrate the characteristics of the electric field enhancement near the particles. In order to compare to the case without the spherical anisotropy, the permittivity of the isotropic counterpart is assumed: $\varepsilon_{i \mathrm{r}}=\varepsilon_{i \mathrm{t}}=14 / 3 \varepsilon_{0}(i=\mathrm{c}$ or s) [2]. In addition, to characterize the anisotropy degree, we keep the simple average $\varepsilon_{i \mathrm{r}} / 3+2 \varepsilon_{i \mathrm{t}} / 3=14 / 3 \varepsilon_{0}$ unchanged, while $\varepsilon_{i \mathrm{r}}\left(\right.$ or $\left.\varepsilon_{i \mathrm{t}}\right)$ is varied.

\subsection{Gold shell with a spherically anisotropic core}

The extinction cross section for a small coated particle consisting of a spherically anisotropic core and a gold shell is shown in Fig. 2. For comparison, we include the results for an isotropic core with comparable isotropic permittivity $\varepsilon_{\mathrm{c}}=\varepsilon_{\mathrm{cr}} / 3+2 \varepsilon_{\mathrm{ct}} / 3$. We find that there exist two dipole plasmon resonances for each extinction spectrum. It is known that one resonant peak at the shorter wavelength $\lambda_{\text {- }}$ results from the antibonding resonance, which is an antisymmetric coupling between the surface charge oscillations on two interfaces at $r=a$ and $r=b$, while the other resonant peak at the longer wavelength $\lambda_{+}$corresponds to symmetric coupling [31]. When spherical anisotropy is taken into account, one observes that the resonant wavelengths for both symmetric and antisymmetric modes exhibit a blue shift in comparison with the isotropic case, resulting in the high surface plasmon resonant energy. Actually, for small coated particles, the quasistatic limit may be valid. In this connection, from the expression of the electric dipole factor (see (6)), the anisotropic core can be re-

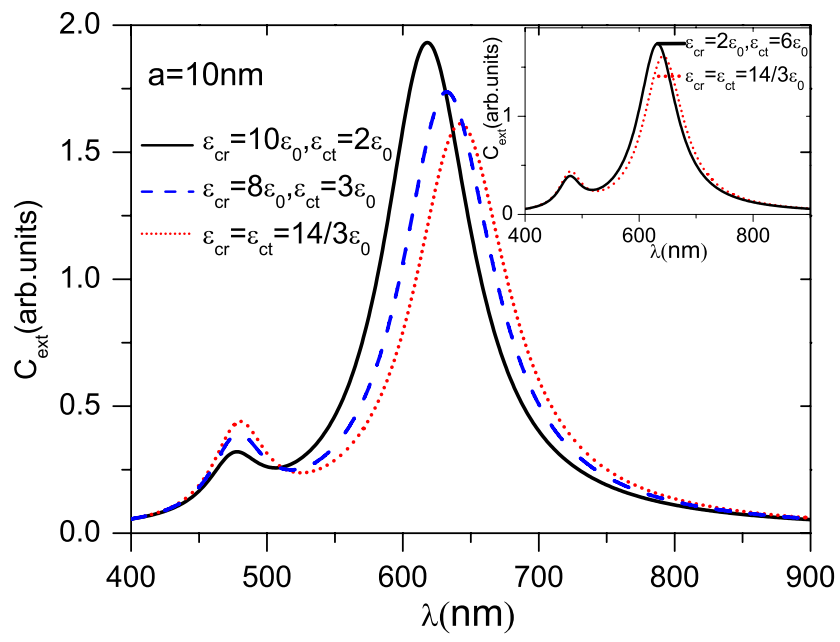

Fig. 2 The extinction spectra of spheres consisting of an Au shell and a spherically anisotropic core for $b=1.5 a$ 


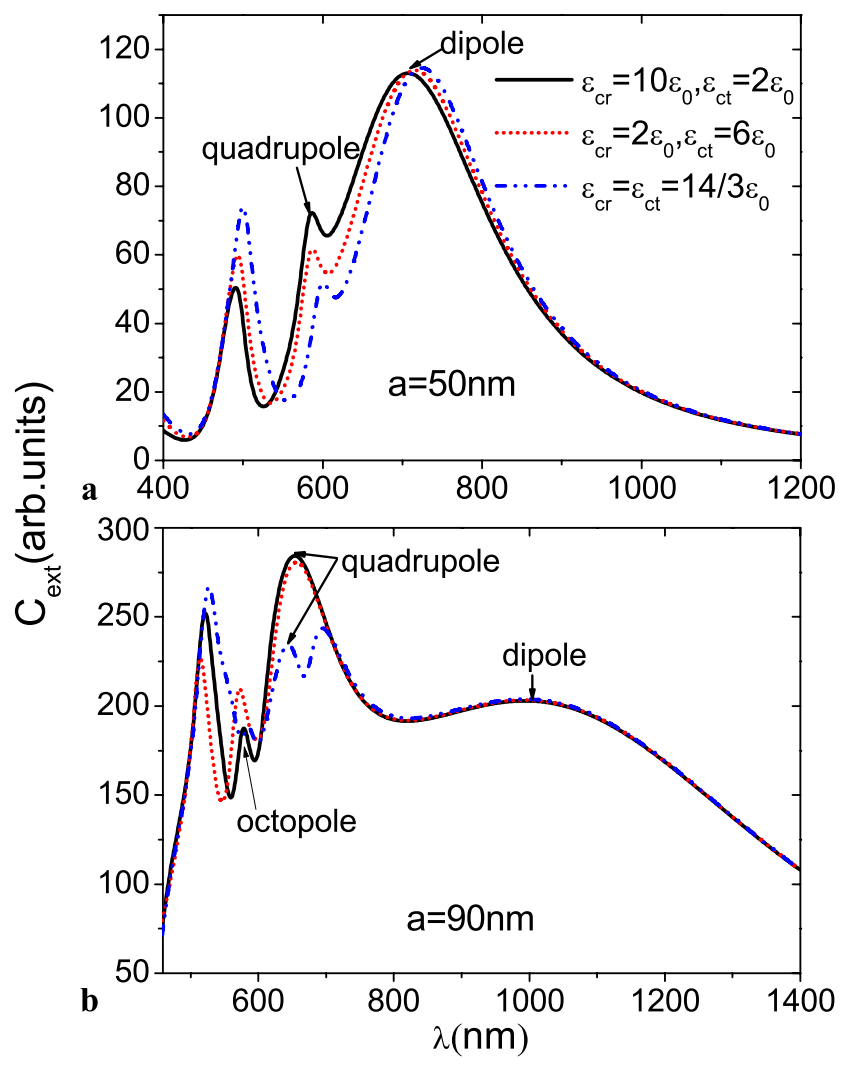

Fig. 3 The extinction spectra of spheres consisting of an Au shell and a spherically anisotropic core for $b=1.5 a$ with (a) $a=50 \mathrm{~nm}$ and (b) $a=90 \mathrm{~nm}$

garded as the isotropic core with the equivalent permittivity $\varepsilon_{\mathrm{c}}=\varepsilon_{\mathrm{cr}} v_{\mathrm{c} 1}^{1}=\sqrt{\left(14 \varepsilon_{0} \varepsilon_{\mathrm{cr}}-3 / 4 \varepsilon_{\mathrm{cr}}^{2}\right)}-1 / 2 \varepsilon_{\mathrm{cr}}$, which is a nonmonotonic function of $\varepsilon_{\mathrm{cr}}$ and has a maximal value $14 \varepsilon_{0} / 3$ when $\varepsilon_{\mathrm{cr}}=\varepsilon_{\mathrm{ct}}$. That is to say, when $\varepsilon_{\mathrm{cr}}$ deviates from the isotropic value $14 / 3 \varepsilon_{0}$, the equivalent permittivity of the anisotropic core is always smaller than that of the isotropic core with $\varepsilon_{\mathrm{c}}=14 / 3 \varepsilon_{0}$, and the introduction of the spherical anisotropy is equivalent to decreasing the core's permittivity. As a consequence, the induced charge on the interface between the core and the shell will be increased, resulting in enhanced restoring forces and large resonant energy. In addition, since the equivalent permittivity for the anisotropic core is smaller than the value for the isotropic core, the strength of the symmetric dipole plasmon resonance should be increased, while the strength of the antisymmetric mode should be decreased when we introduce the spherical anisotropy. Similar conclusions have been made for an isotropic metallic nanoshell based on the time-dependent density functional method [32].

For large nanoshells, the electrostatic approximation may lose its accuracy [2, 27], and we must use full-wave scattering theory to investigate the extinction properties. It is found that there are three resonances in the extinction spectra for large particles with $a=50 \mathrm{~nm}$ and $b=75 \mathrm{~nm}$ (see Fig. 3a), in contrast to two peaks for the small coated particles (see Fig. 2). The additional peak at about $600 \mathrm{~nm}$ on the black solid curve is the contribution from the quadrupole $(n=2)$ resonance of the symmetric mode. Again, we predict blue shifts of both the dipole and quadrupole resonant modes due to the introduction of the spherical anisotropy, as shown in Fig. 3a. However, when the size of the particles is increased further, the resonant wavelength of the dipole $(n=1)$ mode remains unchanged (see Fig. 3b). One further notes that multiple resonances such as octopole modes appear in Fig. 3b because the higher-order scattering dominates the extinction spectra.

In order to study the influence of the spherical anisotropy on the properties of dipole and quadrupole resonant modes clearly, we show the resonant peak positions and strengths of the nanoparticles with different sizes versus $\varepsilon_{\mathrm{cr}}$ in Fig. 4. At first, we discuss the properties for the dipole modes of different sizes (see the left-hand column of Fig. 4). We find that the anisotropy of the core will lead to a blue shift of the resonant wavelength when the radius of the core is smaller than $90 \mathrm{~nm}$ (see Fig. 4a), which is quite similar to the electrostatic case. For instance, on increasing $\varepsilon_{\mathrm{cr}}$ from $\varepsilon_{0}$ to the isotropic permittivity $14 / 3 \varepsilon_{0}$, and then to $10 \varepsilon_{0}$ (i.e. $\varepsilon_{\mathrm{ct}}$ varies from $6.5 \varepsilon_{0}$ to $14 / 3 \varepsilon_{0}$, and to $2 \varepsilon_{0}$ ), the peak position increases from $707 \mathrm{~nm}$ at $\varepsilon_{\mathrm{cr}}=\varepsilon_{0}$ to $725 \mathrm{~nm}$ at $\varepsilon_{\mathrm{cr}}=14 / 3 \varepsilon_{0}$, and then decreases to $708 \mathrm{~nm}$ at $\varepsilon_{\mathrm{cr}}=10 \varepsilon_{0}$. However, when the radius of the core is equal to or larger than $90 \mathrm{~nm}$, the resonant wavelength of the dipole resonance remains unchanged (see Fig. $4 \mathrm{~b}$ and c) with the variation of $\varepsilon_{\mathrm{cr}}$. This is due to the fact that for large nanoparticles, phase retardation becomes important and the extinction results mainly from the electromagnetic scattering not the absorption. As a consequence, the slight variation of the physical properties in the core leads to no obvious shift in the dipole resonant frequency [33]. As far as the peak strength of the dipole mode is concerned, it is decreased when the spherical anisotropy is introduced. The qualitative understanding of the changes of the peak strength of the dipole mode can be similarly understood in terms of plasmon hybridization [31, 32]. Then, we examine the properties of the quadrupole resonant mode (see the right-hand column of Fig. 4). For a not too large nanoshell with $a=50 \mathrm{~nm}$ (see Fig. 4d), the peak position of the quadrupole resonance exhibits a quite similar shift to that of the dipole resonance (see Fig. 4a), but with a much larger shift value. On the other hand, for nanoshells with larger sizes such as $a=90 \mathrm{~nm}$ and $a=110 \mathrm{~nm}$ (see Fig. 4e and $f$ ), the resonant peak of the quadrupole mode exhibits an obvious shift instead of no shift for the dipole mode of larger nanoparticles when we change $\varepsilon_{\mathrm{cr}}$ due to weak coupling to light due to phase retardation. To one's surprise, the peak strengths of the quadrupole resonant modes take on quite different nonmonotonic behaviors dependent on the size of the nanoparticles. 
Fig. 4 (a)-(c) The peak positions and strengths of the dipole resonances of spheres consisting of an Au shell and a spherically anisotropic core with different sizes versus $\varepsilon_{\mathrm{cr}}$. (d) and (e) correspond to the quadrupole modes
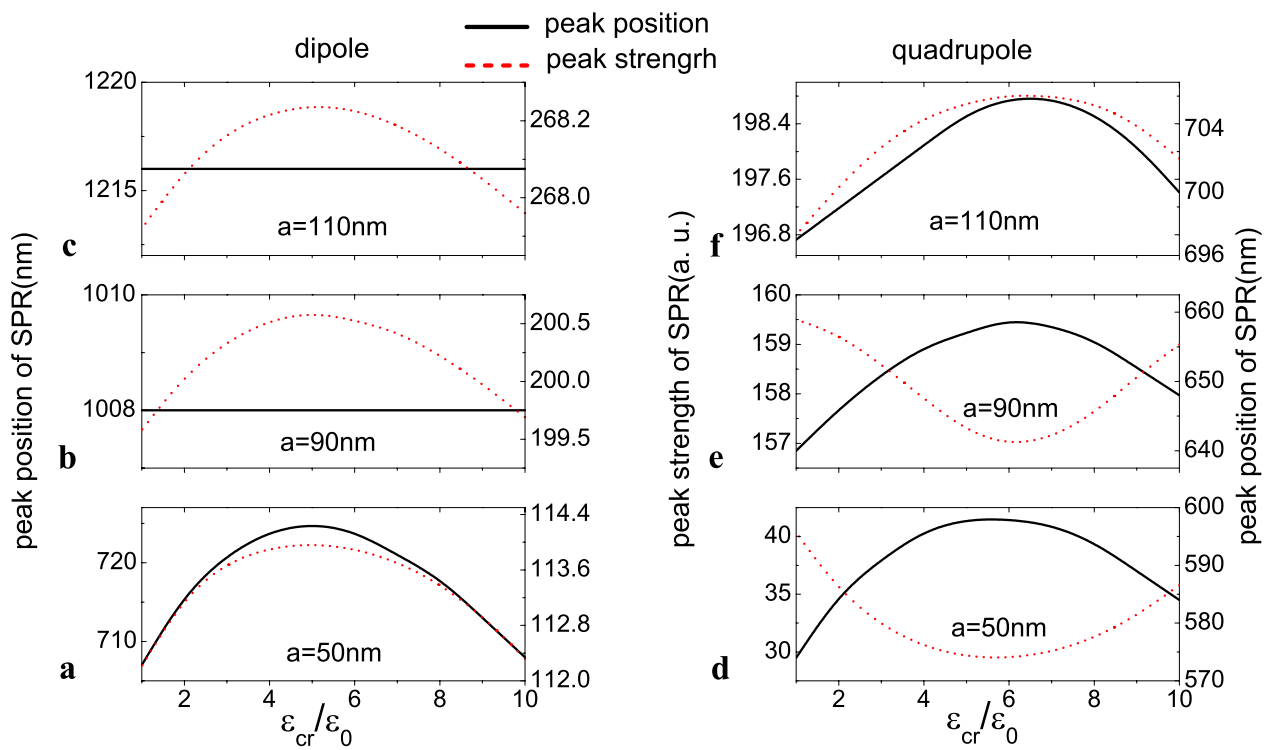

Fig. 5 The electric field enhancement $|\mathbf{E}| /\left|\mathbf{E}_{\mathbf{0}}\right|$ in the $x-y$ plane for a spherically anisotropic core coated by an Au nanoshell for $a=10 \mathrm{~nm}$ and $b=15 \mathrm{~nm}$. Other parameters are $(\mathbf{a}): \varepsilon_{\mathrm{cr}}=10 \varepsilon_{0}, \varepsilon_{\mathrm{ct}}=2 \varepsilon_{0}$, $\lambda=618 \mathrm{~nm} ;(\mathbf{b}): \varepsilon_{\mathrm{cr}}=2 \varepsilon_{0}$, $\varepsilon_{\mathrm{ct}}=6 \varepsilon_{0}, \lambda=632 \mathrm{~nm}$; (c): $\varepsilon_{\mathrm{cr}}=\varepsilon_{\mathrm{ct}}=(14 / 3) \varepsilon_{0}$, $\lambda=642 \mathrm{~nm} ;(\mathbf{d}): \varepsilon_{\mathrm{cr}}=10 \varepsilon_{0}$, $\varepsilon_{\mathrm{ct}}=2 \varepsilon_{0}, \lambda=478 \mathrm{~nm}$
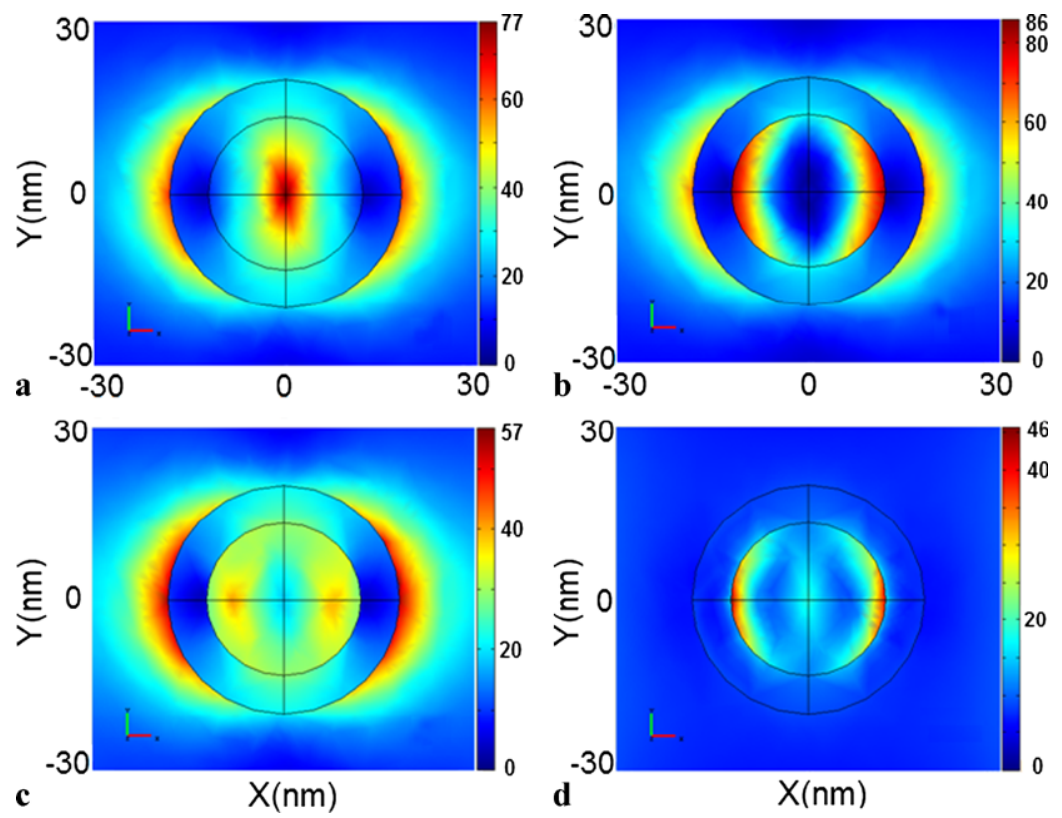

Incidentally, from the comparison of Figs. 2 and 3, it is found that the increase in the size of the core-shell particle results in a red shift of the resonant wavelength of the dipole mode, although the core-shell radius ratio is the same [34]. And, the quadrupole resonance behaves in a similar fashion (it is shown in Fig. 4). Therefore, we conclude that the intensity of the plasmon peaks and the resonant wavelength positions can be tuned through the introduction of the spherical anisotropy and the change in the size of the nanoparticles.

The electric field enhancement in the $x-y$ plane in the vicinity of the anisotropic core-shell particle is presented in Fig. 5. The near-field patterns corresponding to the four cases in Fig. 2 are demonstrated, respectively. It is obvious that the localized fields will be dramatically enhanced at plasmon resonant frequencies. For the symmetric coupling (Fig. 5a-c), along the incident polarization, the enhanced electric fields occur on the outer surface of the gold shell, which clearly demonstrates that the symmetric modes are dominated by the contribution from the plasmon resonance on the outer surface of the nanoshell. Taking the isotropic case (i.e. Fig. 5c) as the reference where the significant field enhancement only exists outside the gold shell, one finds that the spherical anisotropy in the core can actually control the localized field enhancement in the core region while still largely maintaining the enhancement outside the gold shell. For instance, when the spherical anisotropy in the core is given by core, $\varepsilon_{\mathrm{cr}}=10 \varepsilon_{0}, \varepsilon_{\mathrm{ct}}=2 \varepsilon_{0}$ in Fig. 5a, the significant field enhancement occurs to both the center region 

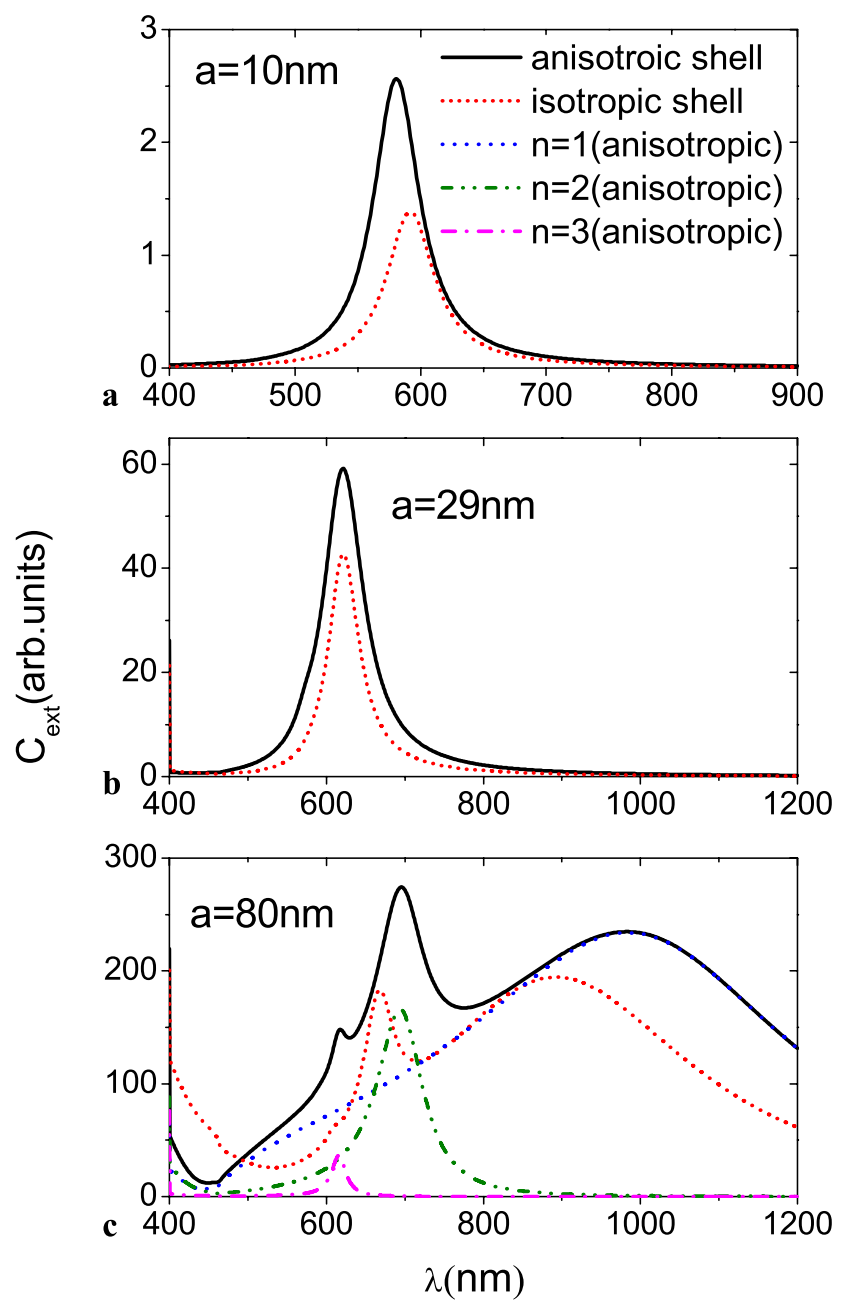

Fig. 6 The extinction spectra of spheres made of an Au core and a spherically anisotropic shell under fixed $b=1.5 a$ for (a) $a=10 \mathrm{~nm}$; (b) $a=29 \mathrm{~nm}$; (c) $a=80 \mathrm{~nm}$. The permittivity of the anisotropic shell is $\varepsilon_{\mathrm{sr}}=10 \varepsilon_{0}, \varepsilon_{\mathrm{st}}=2 \varepsilon_{0}$, and the permittivity of the isotropic shell is $\varepsilon_{\mathrm{sr}}=\varepsilon_{\mathrm{st}}=(14 / 3) \varepsilon_{0}$

of the core and the region outside the shell. On the contrary, when the spherical anisotropy in the core is given by $\varepsilon_{\mathrm{cr}}=2 \varepsilon_{0}, \varepsilon_{\mathrm{ct}}=6 \varepsilon_{0}$ in Fig. 5b, the significant enhancement in the center of the core would be pushed towards the surface of the core, although the enhancement outside the shell is still kept when compared to Fig. 5a. However, for the antisymmetric coupling (Fig. 5d), the significant field enhancement only arises on the inner surface of the gold shell. This indicates that the antisymmetric mode gets its dominant contribution from the (cavity) plasmon on the inner interface. Therefore, the electric field enhancement is tunable by properly controlling the spherical anisotropy of the dielectric core, not only enhancing its maximal value but also varying the location of enhancement region. To one's interest, the magnitude of the local fields can increase from 57 (Fig. 5c) to 86 (Fig. 5b) by introducing a suitable spherical anisotropy. Furthermore, for symmetric modes, it is inter- esting to note that a larger $\varepsilon_{\mathrm{cr}}$ results in a dramatic field enhancement in the center of the core, while a larger $\varepsilon_{\mathrm{ct}}$ leads to a maximal field enhancement on the surface of the core close to the core-shell interface. In addition, based on finiteelement simulation, it can be seen that the electric field inside the isotropic core is not a constant, as suggested from the quasistatic approximation [27].

\subsection{Gold core with a spherically anisotropic shell}

Here we consider the coated particles consisting of an $\mathrm{Au}$ core and a spherically anisotropic shell. In Fig. 6a, we find that there is only one resonant peak since there is only one dielectric-metal interface (at $r=a$ ). The dipole resonance is blue shifted due to the introduction of the anisotropy in the shell. The blue shift for this model can be understood as follows. In the present model, the core is composed of $\mathrm{Au}$ nanoparticles, and it can be embedded in an effective host medium with the permittivity $\varepsilon_{\mathrm{e}}$ containing the anisotropic shell and the background medium. According to the quasistatic theory, the surface plasmon resonant frequency $\omega_{\mathrm{S}}$ is modified to be $\omega_{\mathrm{s}} \approx \omega_{\mathrm{p}} / \sqrt{1+2 \varepsilon_{\mathrm{e}} / \varepsilon_{0}}$. With the introduction of the spherical anisotropy in the shell, the effective permittivity $\varepsilon_{\mathrm{e}}$ for the host medium is decreased, resulting in large $\omega_{\mathrm{s}}$ and small resonant wavelength.

Of particular interest is that when the nanoparticle becomes larger (e.g. the inner radius of the core-shell particle is $80 \mathrm{~nm}$ ), a red shift in the dipole resonant wavelength arises when compared to the isotropic case (see Fig. 6c). This is due to the fact that the dipole resonant energy is related to not only the permittivities of the metal and the spherical anisotropic medium, but also to the size of the coated structure [34]. If the core-shell radius ratio is fixed, increasing the size of the particle will lead to a red shift of the plasmon resonance. On the contrary, the spherical anisotropy will lead to a blue shift of the resonant wavelength. Therefore, it is easy to conjecture a 'neutral' state, i.e. the dipole resonant wavelength exhibits no shift (see Fig. 6b). The size effect will prevail over the spherical anisotropy effect when the core radius is larger than $29 \mathrm{~nm}$. In addition, one can see that multiple resonance modes (see Fig. 6c) appear when $a=80 \mathrm{~nm}$, as expected for larger particles, and the presence of spherical anisotropy in the shell results in an increased peak strength in the extinction spectrum.

\section{Conclusion}

In this paper, we investigated the effect of the spherical anisotropy on surface resonant modes in the extinction spectra and near-field enhancement for both a gold nanoshell with a spherically anisotropic core and a gold nanocore with a spherically anisotropic shell through full-wave scattering 
theory. We found that the resonant wavelengths, the intensity of the extinction peaks and the electric field enhancement can be tailored via controlling the spherical anisotropy introduced into the core-shell system, and the physical insights behind those interesting phenomena are discussed. Moreover, the size effect and the anisotropy effect in controlling the resonant shifts were illustrated. We believe that the current work may be useful for studying the light manipulators, optoelectronics and biomedical identification.

Acknowledgements This work was supported by the National Natural Science Foundation of China under Grant No. 11074183, the Key Project in Natural Science Foundation of Jiangsu Education Committee of China under Grant No. 10KJA140044, the Key Project in Science and Technology Innovation Cultivation Program and the Plan of Dongwu Scholar, Soochow University. Z.F.S. is grateful for support from the Natural Science Foundation of Jiangsu Education Committee of China under Grant No. 07KJD140181.

\section{Appendix}

We give the definition of the symbols in the scattering coefficients.

In (3), we have $k_{i \mathrm{t}}=\omega / c \sqrt{\varepsilon_{i \mathrm{t}}}$. And, $\psi_{n}, \chi_{n}$ and $\varsigma_{n}(x)$ denote the Ricatti-Bessel functions

$$
\begin{aligned}
& \psi_{n}(x)=\sqrt{\frac{\pi x}{2}} J_{n+1 / 2}(x) ; \\
& \chi_{n}(x)=-\sqrt{\frac{\pi x}{2}} N_{n+1 / 2}(x) ; \\
& \varsigma_{n}(x)=\sqrt{\frac{\pi x}{2}} H_{n+1 / 2}^{1}(x),
\end{aligned}
$$

where $J_{n+1 / 2}(x), N_{n+1 / 2}(x)$ and $H_{n+1 / 2}^{1}(x)$ represent the Bessel, Neumann and first-kind Hankel Functions, respectively. The primes on $\psi_{n}, \chi_{n}$ and $\zeta_{n}(x)$ denote differentiations with respect to the argument. The orders of the RicattiBessel functions are defined as follows:

$v_{i 1}^{n}=\sqrt{n(n+1) \frac{\varepsilon_{i \mathrm{t}}}{\varepsilon_{i \mathrm{r}}}+\frac{1}{4}}-\frac{1}{2}, \quad v_{i 2}^{n}=\sqrt{n(n+1)+\frac{1}{4}}-\frac{1}{2}$,

where $i=\mathrm{c}, \mathrm{s}$.

\section{References}

1. C.W. Qiu, L.W. Li, T.S. Yeo, S. Zouhdi, Phys. Rev. E 75, 026609 (2007)

2. D.H. Liu, C. Xu, P.M. Hui, Appl. Phys. Lett. 92, 181901 (2008)

3. S.J. Oldenburg, R.D. Averitt, S.L. Wsetcott, N.J. Halas, Chem. Phys. Lett. 288, 243 (1998)

4. H. Wang, G.P. Goodrish, F. Tam, C. Oubre, P. Nordlander, N.J. Halas, J. Phys. Chem. B 109, 11083 (2005)

5. C. Liu, C.C. Mi, B.Q. Li, IEEE Trans. Nanobiosci. 7, 206 (2008)

6. M. Alam, Y. Massoud, IEEE Trans. Nanotechnol. 5, 265 (2006)

7. S. Deng, K.C. Pingali, D.A. Rockstraw, IEEE Sens. J. 8, 730 (2008)

8. S.J. Oldenburg, G.D. Hale, J.B. Jackson, N.J. Halas, Appl. Phys. Lett. 75, 1063 (1999)

9. C. Oubre, P. Nordlander, J. Phys. Chem. B 108, 17740 (2004)

10. S.J. Oldenburg, J.B. Jackson, S.L. Wsetcott, Appl. Phys. Lett. 75, 2897 (1999)

11. C. Loo, A. Lowery, N. Halas, Nano Lett. 5, 709 (2005)

12. Y.L. Geng, X.B. Wu, L.W. Li, B.R. Guan, IEEE Trans. Antennas Propag. 53, 3982 (2005)

13. L. Gao, T.H. Fung, K.W. Yu, C.W. Qiu, Phys. Rev. E 78, 046609 (2008)

14. B.S. Lukyanchuk, C.W. Qiu, Appl. Phys. A 92, 773 (2008)

15. C.W. Qiu, S. Zouhdi, A. Razek, IEEE Trans. Antennas Propag. 55, 3515 (2007)

16. Y.L. Geng, IET Microw. Antennas Propag. 2, 158 (2008)

17. B.I. Popa, S.A. Cummer, Phys. Rev. A 79, 023806 (2009)

18. C.W. Qiu, L. Gao, J.D. Joannopoulos, M. Soliacic, Laser Photon. Rev. 4, 268 (2010)

19. Y.L. Geng, X.B. Wu, L.W. Li, B.R. Guan, Phys. Rev. E 70, 056609 (2004)

20. J. Roth, M.J. Digman, J. Opt. Soc. Am. 63, 308 (1973)

21. B. Lange, S.R. Aragon, J. Chem. Phys. 92, 4643 (1990)

22. P. Peterlin, S. Svetina, B. Zeks, J. Phys. Condens. Matter 19, 136220 (2007)

23. V.L. Sukhorukov, G. Meedt, M. Kurschner, U. Zimmermann, J. Electrostat. 50, 191 (2001)

24. T. Ambjornsson, G. Mukhopadhyay, S.P. Apell, M. Kall, Phys. Rev. B 73, 085412 (2006)

25. J.B. Pendry, D. Schurig, D.R. Smith, Science 312, 1780 (2006)

26. L. Gao, X.P. Xu, Eur. Phys. J. B 55, 403 (2007)

27. D.J. Wu, X.J. Liu, Appl. Phys. A 94, 537 (2009)

28. C.F. Bohren, D.R. Huffman, Absorption and Scattering of Light by Small Particles (Wiley, New York, 1983)

29. M. Born, E. Wolf, Principles of Optics: Electromagnetic Theory of Propagation, Interference and Diffraction of Light, 7th (expanded) edn. (Cambridge University Press, Cambridge, 1999)

30. R.D. Averitt, S.L. Westcott, N.J. Halas, J. Opt. Soc. Am. B 16, 1824 (1999)

31. E. Prodan, C. Radloff, N.J. Halas, P. Nordlander, Science 302, 419 (2003)

32. E. Prodan, A. Lee, P. Nordlander, Chem. Phys. Lett. 360, 325 (2002)

33. N.K. Grady, N.J. Halas, P. Nordlander, Chem. Phys. Lett. 399, 167 (2004)

34. U. Kreibig, M. Vollmer, Optical Properties of Metal Clusters (Springer, New York, 1995) 